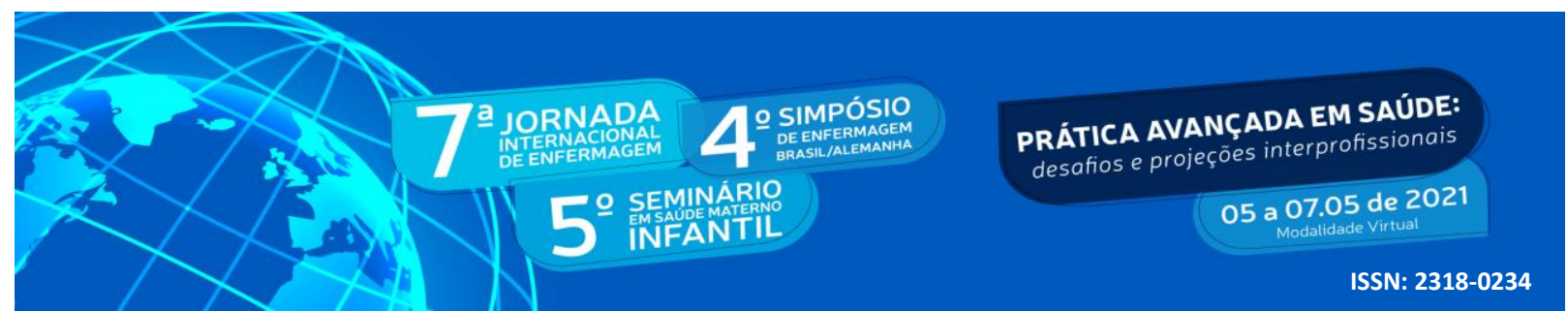

DOI: http://doi.org/10.48195/jie2021-109

\title{
SISTEMATIZAÇÃO DA ASSISTÊNCIA DE ENFERMAGEM E A AUTONOMIA DO ENFERMEIRO
}

\section{Dielli Arend Teixeira1; Pabline Pivetta de Oliveira²; Karen Ariane Bär ${ }^{3}$; Gicelle Moraes Martelli ${ }^{4}$, Graziele Alves Flores ${ }^{5}$; Regina Gema Santini Costenaro ${ }^{6}$}

\section{RESUMO}

O objetivo deste trabalho foi discutir acerca da Sistematização da Assistência de Enfermagem (SAE) como ferramenta de autonomia profissional do enfermeiro. Metodologia: pesquisa do tipo bibliográfica narrativa, foram selecionados cinco (5) artigos, sendo quatro (4) a partir da BVS e somente um (1) da PubMed. Resultados e discussão: emergiram duas categorias, "Sistematização da assistência de Enfermagem como uma metodologia para a prática dos cuidados de enfermagem", e "O cuidado como ferramenta para autonomia e visibilidade dos serviços de enfermagem". Conclusão: Diante aos estudos evidenciados, conclui-se a importância do saber entre a SAE e o PE. Pois o enfermeiro tem a frente nestes requisitos para qualificar seus serviços de saúde, e entender que este processo qualifica seus cuidados de enfermagem com o usuário.

\footnotetext{
1 Estudante do Curso de Enfermagem. Bolsista PROBIC-Universidade Franciscana-UFN- Santa Maria-RS. Email: dielliarend@gmail.com

${ }^{2}$ Estudante do Curso de enfermagem. Bolsista PROBIC- Universidade Franciscana- UFN. Santa Maria-RS. Email: pablinepivetta@gmail.com

${ }^{3}$ Enfermeira. Aluna do mestrado profissional em saúde materna e infantil. Universidade Franciscana-UFN. Santa Maria - RS. Email: bkarenarianebar@gmail.com

${ }^{4}$ Estudante do Curso de enfermagem. Bolsista voluntária. Universidade Franciscana- UFN. Santa Maria-RS. Email: gicelle.martelli@hotmail.com

${ }_{5}^{5}$ Estudante do Curso de enfermagem. Bolsista FAPERGS-PPSUS. Universidade Franciscana- UFN. Santa MariaRS. Email: flores.graziele8@gmail.com

${ }^{6}$ Orientador. Regina Gema Santini Costenaro Enfermeira. Dra, Professora na Universidade Franciscana- UFN. Santa Maria-RS. Email: reginacostenaro@gmail.com
} 


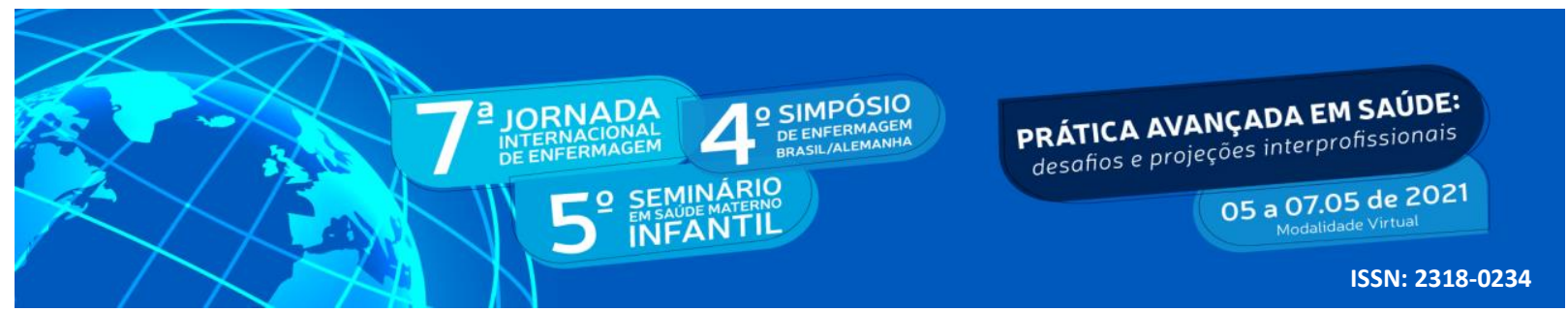

Palavras-chave: Sistematização de Assistência de Enfermagem, Autonomia do enfermeiro, processo em enfermagem.

\section{ABSTRACT}

The objective of this work was to discuss about the Systematization of Nursing Care (SAE) as a tool of professional autonomy of nurses. Methodology: narrative bibliographic research, five (5) articles were selected, four (4) from the VHL and only one (1) from PubMed. Results and discussion: two categories emerged, "Systematization of Nursing care as a methodology for the practice of nursing care", and "Care as a tool for autonomy and visibility of nursing services'. Conclusion: In view of the studies evidenced, the importance of knowledge between NCS and the EP is concluded. Because nurses have the lead in these requirements to qualify their health services, and understand that this process qualifies their nursing care with the user.

Key Words: and understand that this process qualifies their nursing care with the user.

\section{INTRODUÇÃO}

A Sistematização da Assistência de Enfermagem (SAE) é o método empregado para organizar e ampliar as ações dessa equipe de profissionais nos serviços de saúde em geral. A literatura científica descreve que a SAE organiza as informações, análise, interpretação e avaliação dos dados, para tornar possível a operacionalização do Processo de Enfermagem (PE), tendo como objetivo a redução de complicações (ALENCAR, 2018).

No contexto da enfermagem brasileira, a Resolução 358/2009 dispõe sobre a SAE e a implantação do PE em ambientes públicos e privados, em que ocorra o cuidado de enfermagem. Considera que a SAE "organiza o trabalho profissional quanto ao método, pessoal e instrumento, tornando possível a organização do Processo de Enfermagem (PE)" (COFEN,2016). Ainda, explicita que, as disposições da Lei 7.498/86 que regulamenta o Exercício Profissional, é de competência do enfermeiro a liderança na execução e avaliação do PE, cabendo-lhe privativamente o diagnóstico e a prescrição acerca das respostas em um dado momento do processo saúde e doença. Igualmente destaca que os profissionais de enfermagem almejam desenvolver habilidades técnico-científicas que favorecem a organização e sistematização do cuidado (COFEN,2016). 


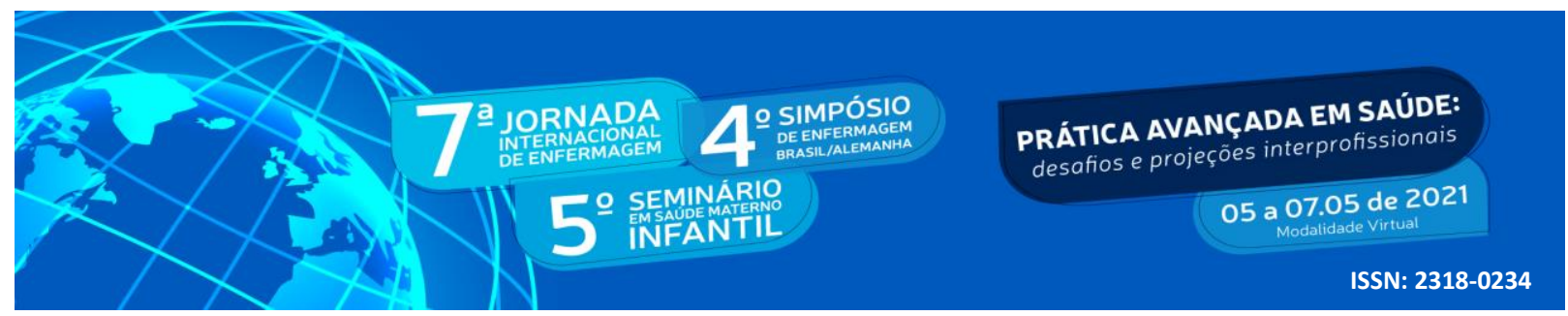

A utilização dos princípios da SAE é uma obrigatoriedade no processo de trabalho do enfermeiro não somente pela sua concepção legal, mas também de ordem ética. Não se pode adiar uma assistência de enfermagem que engloba elementos como: diagnósticos, ações, intervenções e resultados de enfermagem. Assim, sistematizar todo o cuidado e, viabilizar o PE, é uma meta de planejamento e implantação das ações de cuidados de tecnologia e conhecimento científico (OLIVEIRA, 2019).

Frente ao exposto, justifica-se a relevância deste estudo pelas contribuições que esta temática pode acrescentar, de maneira organizada, na prática profissional dos cuidados de enfermagem. Como parte do Processo de Enfermagem está o Histórico de Enfermagem o qual possibilita conhecer a situação do paciente e a partir desta, realizar um plano de cuidados individualizado que contribua para melhorar a qualidade da assistência, baseado nas necessidades específicas da pessoa quee stá sendo cuidada.

\section{OBJETIVO}

Discutir acerca da Sistematização da Assistência de Enfermagem como ferramenta de autonomia profissional do enfermeiro.

\section{METODOLOGIA}

Para atender o objetivo deste estudo, foi realizada uma pesquisa do tipo bibliográfica narrativa. Para tal, foi pesquisado nas bases de dados Biblioteca Virtual em Saúde (BVS) e National Library of Medicine (PubMed) com os descritores em ciências da saúde (DesCS): "Sistematização de Assistência de Enfermagem" and "Autonomia do enfermeiro" and" Processo de enfermagem". A busca aconteceu no mês de março de 2021.

$\mathrm{Na}$ busca de publicações nas bases de dados foram encontrados 38 resultados no total, sendo treze (13) da base de dados BVS e vinte e cinco (25) da PubMed, como critérios de inclusão foram: texto completo, idioma português, recorte temporal de dez anos. Como critérios de exclusão, artigos que não faziam parte do ojetivo proposto, pesquisas do tipo revisões e que não se encaixavam nos critérios de seleção 


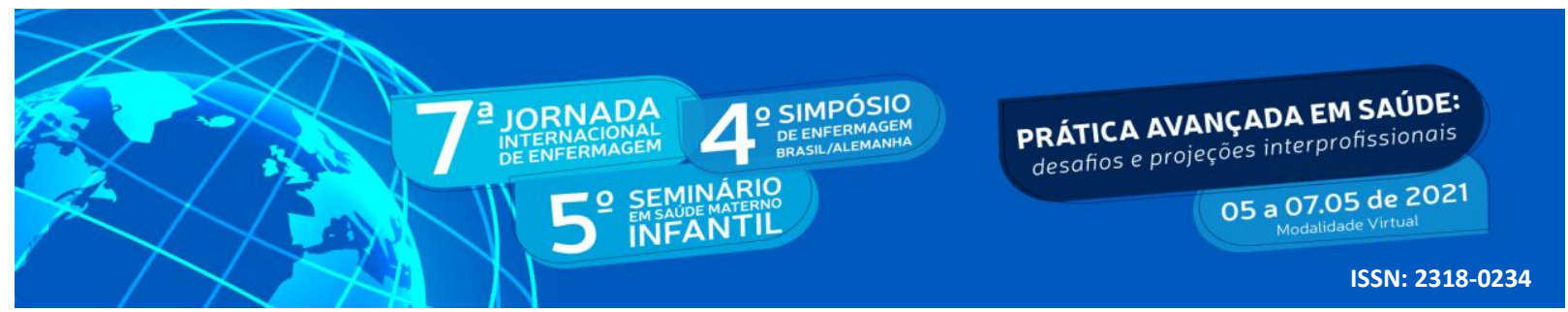

desta busca, assim ao analisar as publicações foram selecionados cinco (5) artigos, sendo quatro (4) a partir da BVS e somente um (1) da PubMed, estes que foram de encontro com o objetivo do estudo.

\section{RESULTADOS E DISCUSSÃO}

A partir da análise e leitura das publicações selecionadas emergiram duas categorias, "Sistematização da assistência de Enfermagem como uma metodologia para a prática dos cuidados de enfermagem", e "O cuidado como ferramenta para autonomia e visibilidade dos serviços de enfermagem".

\subsection{Sistematização da assistência de Enfermagem como uma metodologia para a prática dos cuidados de enfermagem}

O PE é estudado e aplicado nos serviços de saúde no Brasil e no mundo. De acordo com o diagnóstico de enfermagem da Associação Norte-Americana de Diagnósticos de Enfermagem (NANDA), este processo compreende as seguintes etapas: coleta de dados, diagnóstico de enfermagem, planejamento, estabelecimento de resultados, intervenção e avaliação. Os profissionais de enfermagem utilizam a coleta de dados e o julgamento clínico para formular hipóteses ou para explicar acontecimentos reais existentes e ou possibilidades para promoção da saúde (HERDMAN; KAMITSURU, 2015).

Diante disso, Gonçalves; Spiri; Ortolan (2017) relatam que a SAE é o conjunto de fatores científicos e assistenciais prestados antes de realizar alguma conduta, compreendendo também que o processo de enfermagem é uma ferramenta de realizar o cuidado prestado na assistência de enfermagem. Assim, é necessário a existÊncia da SAE para realizar o PE, pois ambas as partes necessitam uma da outra para serem implementadas no serviço de saúde, e o enfermeiro utilizar de sua autonomia quanto a realização da SAE para qualificar seus serviços de saúde, não somente robotizar estes instrumentos, mas sim aprimorar ainda mais seus cuidados (SAMPAIO, 2019). 


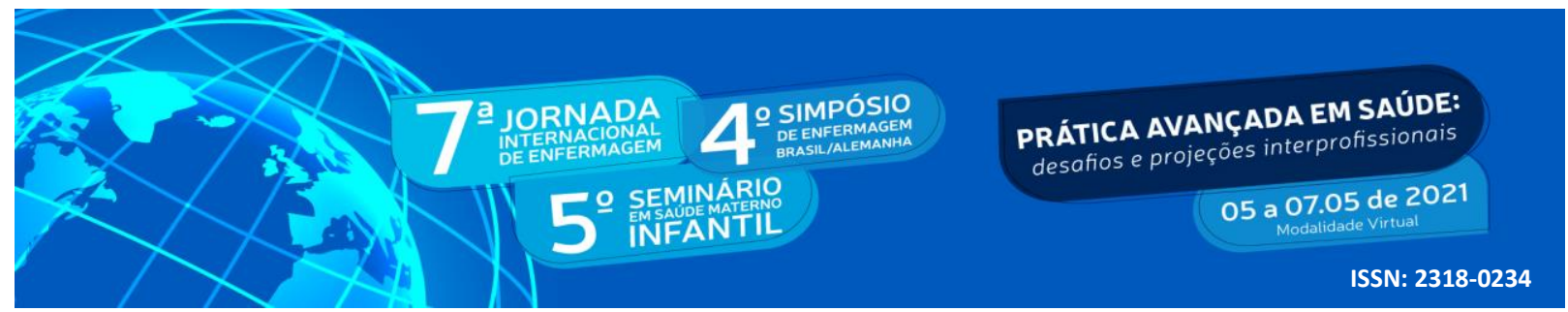

No Brasil, o Processo de Enfermagem, teve sua gênese em 1979, ocasião em que uma renomada enfermeira, Wanda de Aguiar Horta, ancorada nas ideias da teoria de Maslow, vislumbrou uma assistência de enfermagem embasada nas Necessidades Humanas Básicas. A referida teoria tem como base, que todos os seres humanos possuem as mesmas necessidades para manter sua existência. Estas são descritas da seguinte forma: Necessidades fisiológicas, Necessidades de segurança, necessidades sociais, Necessidades psicológicas (estima, amor e relacionamento), necessidades de autorrealização pessoal (solução de problemas, ausência de preconceito, valorização espiritual) (POTTER; PERRY, 2007).

O estudo sobre a SAE no Brasil destacou-se somente no final de 1980, quando o Decreto n 94.406/87, que regulamenta a Lei 7.498/86 do Exercício Profissional de Enfermagem no país, reforçado pela Resolução 358/2009, do Conselho Federal de Enfermagem (COFEN), que dispõe sobre a implementação da SAE. Deste modo, julga que a mesma organiza o trabalho do profissional diante do método, sua equipe e dos instrumentos, operacionalizando o Processo de Enfermagem, a fim de certificar a qualidade na assistência humana em todo o ciclo vital (COFEN, 2009).

A SAE tem como principal propósito induzir um pensamento crítico-reflexivo para a prática de enfermagem e, dessa forma, nortear a tomada de decisões com base em evidências científicas (ARAÚJO, 2016). Sistematizada por meio do PE, a SAE proporciona a organização do cuidado e permite ampliar conhecimentos teóricos à prática. Sua execução fortalece a Enfermagem como ciência e torna a atuação do Enfermeiro mais resolutiva, eficiente e autônoma no conjunto das atribuições multiprofissionais (SANTOS; LIMA; MELO, 2014).

Nessa mesma direção, a SAE possibilita a identificação das demandas de forma contextualizada, isto é, a partir das necessidades singulares de cada pessoa, família e comunidade, bem como o direcionamento das intervenções de enfermagem de forma proativa, criativa e integrada à atuação dos demais profissionais da equipe multiprofissional (MARIA; QUADROS; GRASSI, 2012).

Do mesmo modo, Sampaio (2019) afirma que o trabalho do enfermeiro quando organizado através do PE, das evoluções completas e exame físico efetuados pelos 


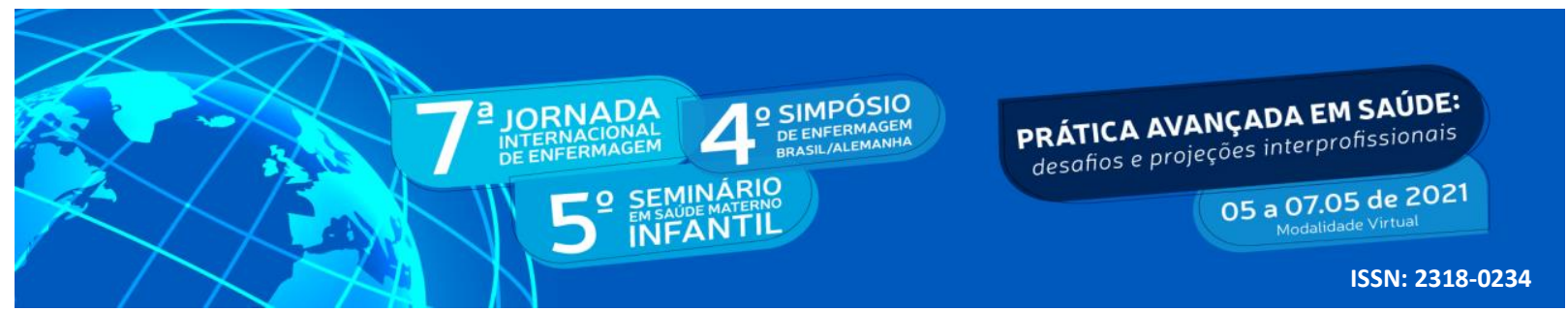

enfermeiros adequadamente, é compreendido como uma prática de enfermagem com subsídios científicos para estabelecer e executar diferentes linhas de cuidados a serem implementados à equipe de enfermagem de acordo com as demandas de cuidado que o paciente necessita, e não apenas um cuidado pautado no apoio à prática profissional médica.

Assim, além de orientar a prática de enfermagem, a SAE visa transcender a 16 fragmentação do cuidado acrítico e mecanizado, frequentemente conduzido pelos profissionais de enfermagem de forma rotineira e verticalizada. O Histórico de Enfermagem (HE) é considerado fase propulsora para as demais etapas do Processo de Enfermagem. Deve ser desenvolvido por meio da coleta de dados e tem como técnicas de investigação mais utilizadas, a entrevista singularizada com o usuário e a sua família, a observação participante e o exame físico (MENEZES; PRIEL; PEREIRA, 2011; NUNES et al., 2011). Realizado de forma minuciosa e clinicamente competente, - Histórico de Enfermagem conduzirá a resultados mais efetivos, eficazes e resolutivos no processamento da sistematização da assistência (SANTOS; VEIGA; ANDRADE, 2011).

Com base em um raciocínio clínico, o Histórico de Enfermagem possibilitará a identificação de necessidades tanto de ordem biológica e psicológica quanto de ordem social e espiritual, as quais subsidiarão a elaboração e a implementação do plano de cuidados na perspectiva da Teoria das Necessidades Humanas Básicas de Wanda Horta (DOMINGOS et al., 2015; MENEZES; PRIEL; PEREIRA, 2011). O plano de cuidados coerente e consistente possibilitará, por sua vez, intervenções teoricamente sustentadas e condizentes com as reais necessidades dos usuários (SANTOS; VEIGA; ANDRADE, 2011).

\subsection{O cuidado como ferramenta para autonomia e visibilidade dos serviços de enfermagem}

O cuidado compreende a linguagem da enfermagem e, ao visualizá-lo como um modo de ser, relacional e contextual, caracteriza-se como a única ação verdadeiramente independente do enfermeiro. Para verificar a autonomia profissional, o ato de cuidar deve ser organizado de forma a respeitar a singularidade da 


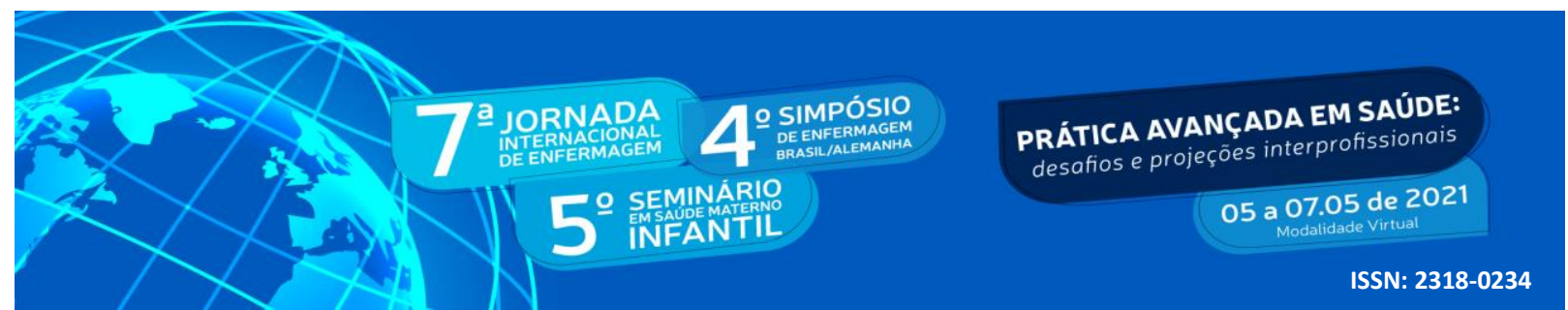

experiência do adoecer e viver, e significar um conjunto de relações e interações entre os indivíduos envolvidos no processo, além de se fundamentar na rigorosidade da sistematização (FERREIRA et al., 2016).

Nesse âmbito, a SAE revela-se como um formidável instrumento metodológico que admite o desempenho dos profissionais de enfermagem no processo de cuidar fortalecendo o vínculo com os pacientes, tanto na elaboração quanto na prestação do cuidado, possibilita o reconhecimento da qualidade de seu trabalho junto ao paciente, refletindo na conquista de autonomia e tomada de decisão do enfermeiro (SANTOS; MONTEZELI; PERES, 2012; MENEZES, PRIEL, PEREIRA, 2011).

Todavia, observa-se que a SAE não é realizada em muitos serviços de saúde, sendo justificada pela grande demanda, reduzido número de profissionais, ausência de prontuários, de recursos materiais e de equipamentos. Embora seja considerada pelos enfermeiros como uma prática que contribui para a conquista de seu papel como integrante da equipe de saúde, proporcionando autonomia, reconhecimento e valorização profissional (NUNES et al., 2011; SANTOS et al., 2011).

A autonomia envolve a competência humana em acompanhar suas normas, bem como, exercer a capacidade de liberdade nas suas condutas de acordo com a condição vivenciada e proporcionada pela valorização do seu trabalho (BIANCO, 2000). Nesse sentido, os enfermeiros julgam o gerenciamento da assistência como a prática mais relevante para o desenvolvimento da autonomia profissional (SAMPAIO, 2019).

No entanto, a autonomia é conquistada por aqueles que assumem o papel na transformação da sua prática a partir das relações de poder estabelecidas com sua equipe na perspectiva do crescimento conjunto. Independente da unidade hospitalar, os profissionais de enfermagem passam pelas mesmas situações de trabalho e precisam desenvolvê-lo com conhecimento e eficácia, sempre garantindo a individualidade, integralidade e suporte de qualidade na assistência ao paciente (SANTOS, 2016; MENEZES, PRIEL, PEREIRA, 2011).

Portanto, percebe-se que a autonomia possui importante relação com 0 conhecimento profissional e seus saberes. Os profissionais da enfermagem que 


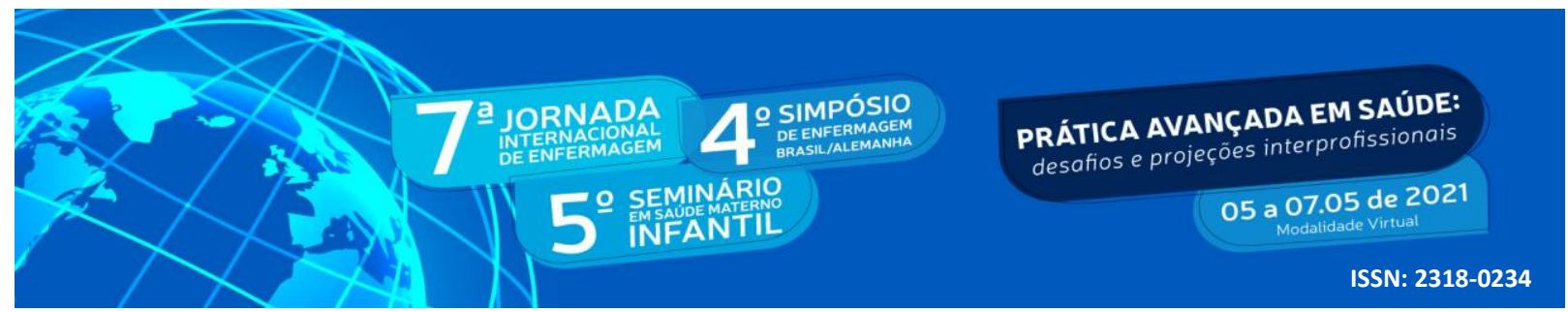

almejam o aprimoramento pessoal e profissional devem participar de espaços educativos, contribuindo assim, para o aprimoramento do trabalho e consequente qualificação do cuidado que presta. Essas atitudes fortalecem a visibilidade das ações de enfermagem e concomitantemente, oportunizam uma significativa autonomia profissional (OLIVEIRA, 2012; SANTOS et al., 2011; SOUZA, 2013).

Estas evidências sobre a SAE e o PE, são de grande valia para o entendimento da grande importância que o enfermeiro tem dentro do serviço de saúde. Buscando com isso sempre o aprimoramento destes conceitos e de suas condutas para melhor resultar o cuidado com seu usuário. Lembrando também que a utilização dessas ferramentas, possibilita a customização do tempo dos profissionais da saúde e uma visão mais ampliada sobre os recursos que possam ser utilizados na implementação da SAE.

\section{CONCLUSÃO}

Diante aos estudos evidenciados, conclui-se a importância do saber entre a SAE e o PE. Pois o enfermeiro tem a frente nestes requisitos para qualificar seus serviços de saúde, e entender que este processo é de grande valia para a enfatização de sua autonomia quando profissional enfermeiro. Entender toda a contextualização da SAE, possibilita que o enfermeiro conduza melhor sua equipe para melhor realização de todo o $\mathrm{PE}$.

Entretanto, o cuidado de enfermagem está presente como um dos fatores de autonomia da equipe de enfermagem. Pois o aprimoramento deste serviço possibilita um cuidado singular e adequado para cada usuário, compreendendo suas necessidades e especificidades. Assim, o enfermeiro deve entender o conjunto de ações entre o processo para chegar até os resultados esperados.

Contudo, ainda é visualizado a fragilidade no entendimento da importância do $\mathrm{PE}$, pois muitas vezes é evidenciado a robotização do serviço de saúde, e o não o conhecimento científico sobre a importância da implementação e prestação dos cuidados. Saber oque e por que a equipe está realizando determinado cuidado. Por isso este conhecimento deve ser compartilhado nos espaços de saúde, e por meio da 


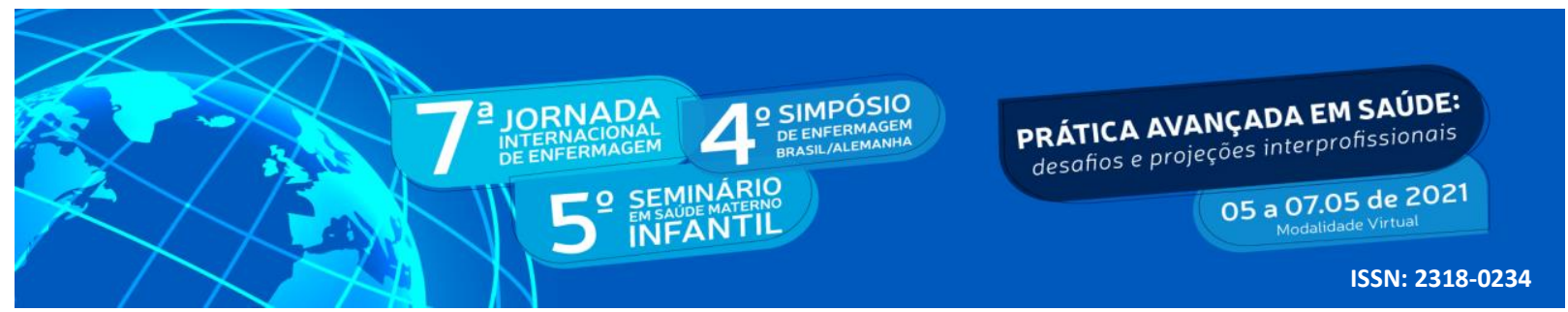

operacionalização do PE o enfermeiro exerce e visibiliza sua autonomia profissional, além de customizar seu tempo e da equipe nos cuidados realizados.

\section{REFERÊNCIAS}

ALENCAR, I. G. M.; NUNES, V. S.; ALVES, A. S.; LIMA, S. L. R.; MELO, G. K. M.; SANTOS, M. A. F. Implementação e implantação da sistematização da assistência de enfermagem. Revista de Enfermagem UFPE online, v.12, n.4, p.1174-8, 2018. ARAÚJO, D. D. Sistematização da assistência de enfermagem e Processo de Enfermagem: aspectos conceituais. Revista Norte Mineira de Enfermagem RENOME, v. 5, n. 1, p. 1-4, 2016.

BIANCO, M. H. B. Construção da autonomia da enfermeira no cotidiano: um estudo etnográfico sob o referencial teórico de Agnes Heller. Bauru: Cadernos de Divulgação e Cultura; 2000.

COFEN. Conselho Federal de Enfermagem. Guia de recomendações para registro de enfermagem no prontuário do paciente e outros documentos de enfermagem. Brasília, 2016.

COFEN. Conselho Federal de Enfermagem (BR). Resolução COFEN 358/2009.

Dispõe sobre a Sistematização da Assistência de Enfermagem e a implementação do Processo de Enfermagem em ambientes, públicos ou privados, em que ocorre o cuidado profissional de Enfermagem, e dá outras providências. Brasília (DF): COFEN; 2009.

DOMINGOS, C. S.; et al. Construção e validação de conteúdo do histórico de enfermagem guiado pelo referencial de OREM. Revista Mineira de Enfermagem, v. 19, n. 2, p. 165-186, 2015.

FERREIRA, E. B.; PEREIRA, M. S.; SOUZA, A. C.; TALEB, A. C. Systematization Of Nursing Care in the perspective of professional autonomy. Revista Rene, v.17, n.1, p.86-92, 2016.

GONÇALVES, M. R. C B.; SPIRI, W. C.; ORTOLAN, E. V P. Sentimento dos enfermeiros de um hospital universitário quanto à prática diária do processo de enfermagem. Revista de Enfermagem UFPE, v.11, n. 5, p.1902-8, 2017. HERDMAN, T. H.; KAMITSURU, S. Diagnósticos de enfermagem da NANDA: definições e classificação 2015-2017. In: Diagnósticos de enfermagem da Nanda: 


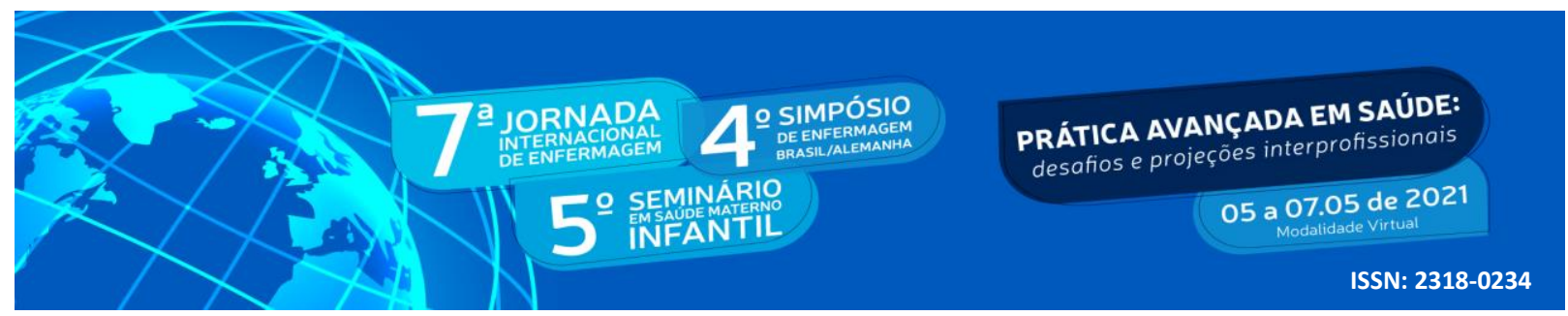

definições e classificação 2015-2017. 2015. p. 468-468.MARIA, M. A.; QUADROS, F. A. A.; GRASSI, M. F. O.. Sistematização da assistência de enfermagem em serviços de urgência e emergência: viabilidade de implantação. Revista Brasileira de Enfermagem, v. 65, n. 2, p. 297-303, 2012.

MENEZES, S. R. T.; PRIEL, M. R.; PEREIRA, L. L. Autonomia e vulnerabilidade do enfermeiro na prática da Sistematização da Assistência de Enfermagem. Revista da Escola de Enfermagem da U S P, v.45, n.4, p.953-8, 2011. NUNES, L. C. B.; SANTOS, V. E. P.; SILVA, A. M. P.; SANTOS, A. L. S.; CARVALHO, M. F. A. A. Sistematização da assistência de enfermagem: aplicação sob ótica dos enfermeiros. Revista de Enfermagem UFPE on line, v.5, n.3, p.55360, 2011.

OLIVEIRA, C. R. Cateter central de inserção periférica em neonatologia e pediatria: as vozes das enfermeiras [Dissertação]. Santa Maria: Universidade Federal de Santa Maria; 2012.

OLIVEIRA, M. R.; ALMEIDA, P. C.; MOREIRA, T. M. M.; TORRES, R. A. M. Nursing care systematization: perceptions and knowledge of the Brazilian nursing. Revista Brasileira de Enfermagem, v.72, n.6, p.1547-53, 2019 POTTER, P.; PERRY, A. G. Hierarquia das necessidades de Maslow. Grande Tratado de Enfermagem Prática e Clínica Hospitalar. 3ª Edição. São Paulo: Editora Santos. 2007.

SAMPAIO, R. S. Contribuições do processo de enfermagem e da sistematização da assistência para a autonomia do enfermeiro. Revista Cubana de Enfermería, v.35, n.2, e177, 2019.

SANTOS, C. P. A adaptação do enfermeiro à cultura organizacional: uma imersão nas formas de produção de subjetividades [Tese]. Rio Grande: Universidade Federal do Rio Grande; 2016.

SANTOS, F. O. F.; MONTEZELI, J. H.; PERES, A. M. Autonomia profissional e sistematização da assistência de enfermagem: percepção de enfermeiros. Revista Mineira de Enfermagem, v.16, n.2, p.251-257, 2012.

SANTOS, J. S.; LIMA, L. M.; MELO, I. A. Sistematização da Assistência de Enfermagem na Unidade de Terapia Intensiva: Revisão Bibliográfica. Ciências Biológicas e da Saúde, v. 2, p. 59-68, 2014.SANTOS, N.; VEIGA, P.; ANDRADE, R. Importância da anamnese e do exame físico para o cuidado do enfermeiro. Revista Brasileira de Enfermagem, v. 64, n. 2, p. 355-358, 2011.

SANTOS, A. L. S.; SANTOS, R. A. A.; SILVA, A. M. P.; LEAL, J. D.; SANTOS, V. E. $P$. Sistematização da assistência de enfermagem: no caleidoscópio dos enfermeiros de um hospital de ensino. Revista de Enfermagem UFPE on line, v.5, n.7, p.1613618, 2011. 